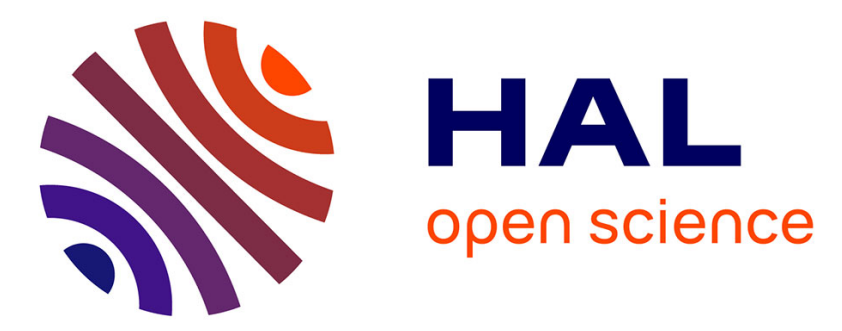

\title{
Effects of orientation, lattice defects and temperature on plasticity and phase transition in ramp-compressed single crystal iron
}

Nourou Amadou, Thibaut de Resseguier, A. Dragon, E. Brambrink

\section{- To cite this version:}

Nourou Amadou, Thibaut de Resseguier, A. Dragon, E. Brambrink. Effects of orientation, lattice defects and temperature on plasticity and phase transition in ramp-compressed single crystal iron. Computational Materials Science, 2020, 172, pp.109318. 10.1016/j.commatsci.2019.109318 . hal03079630

\section{HAL Id: hal-03079630 \\ https://hal.science/hal-03079630}

Submitted on 17 Dec 2020

HAL is a multi-disciplinary open access archive for the deposit and dissemination of scientific research documents, whether they are published or not. The documents may come from teaching and research institutions in France or abroad, or from public or private research centers.
L'archive ouverte pluridisciplinaire HAL, est destinée au dépôt et à la diffusion de documents scientifiques de niveau recherche, publiés ou non, émanant des établissements d'enseignement et de recherche français ou étrangers, des laboratoires publics ou privés. 


\title{
Effects of orientation, lattice defects and temperature on plasticity and phase transition in ramp-compressed single crystal iron
}

\author{
N. Amadou ${ }^{1,2}$, T. de Resseguier ${ }^{1}$, A. Dragon ${ }^{1}$ and E. Brambrink ${ }^{3}$ \\ ${ }^{1}$ Institut Pprime, CNRS, ENSMA, Université de Poitiers, Poitiers, France \\ ${ }^{2}$ Département de Physique, Université Abdou Moumouni de Niamey, BP. 10662 Niamey, Niger \\ ${ }^{3}$ European XFEL GmbH, 22869 Schenefeld, Germany
}

\begin{abstract}
Molecular dynamics simulations have been used to investigate both deformation and bcc-hcp structural phase transition in single crystal iron under ramp compression as function of orientation, initial defects and temperature conditions using the recently developed modified version of the Ackland iron potential. At initial temperature of $50 \mathrm{~K}$, significant anisotropy is observed in the deformation in the form of twinning followed by an elastic stiffening effect in [001] direction, elastic deformation in [111] direction, while [110] direction exhibits the most outstanding feature characterized by yielding via simultaneous twinning and massive homogeneous nucleation of hcp phase resulting in a metastable phase mixture where dislocations develop. However, after introducing microvoid defects in the initial lattice, plastic deformation is observed in all the three directions. Plasticity is mainly mediated by dislocations activities except for the [001] direction where dislocations kinetics is found to be very slow and the plasticity remains dominated by twinning. When the initial temperature is increased above $200 \mathrm{~K}$, twinning is no longer observed, while dislocation density and velocity tend to increase. This dependence of elastic-plastic history on loading direction and initial conditions is found to strongly affect subsequent phase transition.
\end{abstract}

Keywords: iron, plasticity, phase transition, twins, dislocations, nucleation and growth, MD simulations, ramp compression, shock wave, microvoid defects

\section{Introduction}

The dynamic behavior of materials is an area of study at the confluence of many scientific disciplines[1, 2], motivated by both fundamental and industrial applications. Of special importance is understanding of (i) the deformation (specifically plasticity) and phase transitions mechanisms of solid matter under extreme conditions of pressure, temperature and strain rate, (ii) how deformation path and mode affect the structural phase transformations and (iii) how initial microstructure and temperature influence the overall deformation process[3, 4, 5, 6, 7, 2].

During dynamic compression by a planar wave front, material is uniaxially compressed which involves a large deviatoric stress[8]. In practice, such compression is achieved by shock loading[9, 3], where strain rates can reach $10^{9} s^{-1}[10]$. However, shock wave is a highly dissipative process, which causes an entropy jump and high temperatures so that materials may melt[4]. In order to preserve the solid state, the so-called "quasi-isentropic" ramp compression (where the pressure pulse is shaped to rise more progressively over time) is alternatively used [11]. In such approach, strain rates greater than $10^{6} \mathrm{~s}^{-1}$ are currently available[12, 10, 13, 14, 15, 16]. They allow studying the kinetics of both plasticity and phase transitions in solid state[7, 14, 16]. At moderate deformation rates, it is the emission, multiplication and propagation of dislocations that are responsible for plastic deformation where the process can be either thermally activated or limited by dissipation involving phonon emission and scattering $[4,5]$. At high deformation rates, it is rather twinning that dominates and accommodates the high shear stress more rapidly[7]. Furthermore, although isokinetics regime was reported for 
the iron bcc-hcp phase transformation in a narrow range of deformation rates[15], the kinetics of structural transformations remains largely dependent on the strain rate[14, 16]. Understanding these various physical phenomena motivates meso-scale energy-based analyses[17] and purposeful atomic level investigations wherein Molecular Dynamics (MD) [18, 19, 20, 21].

In this context, using the recently developed modified version of the Ackland iron potential[22], we have reported in a previous paper[23] that defect-free single crystal iron of $50 \mathrm{~K}$ initial temperature subjected to ramp compression along the [001] direction first yields by twinning at a longitudinal pressure of $\sim 12 \mathrm{GPa}$, then upon further compression, the twins recede progressively while no dislocation activity is detected. This leads to a new increase of the deviatoric stress to values as high as 30 GPa. Such elastic stiffening of the bcc matrix is shown to inhibit the nucleation of the hcp phase, so that the onset of the phase transformation is shifted to very high pressures (on the order of $100 \mathrm{GPa}$ ). In the present work, we go further to explore both deformation and bcc-hcp structural phase transition and their coupling under ramp compression in other low-index crystallographic directions, in crystals with or without microvoid defects, with different initial temperature conditions. The deformation behavior is found to be highly anisotropic in both defect-free and defective single crystal. At low temperature, plastic deformation, strongly enhanced by the presence of initial defects, is mainly dominated either by twinning alone or twinning and dislocations activities in different proportions, depending on loading direction with respect to crystal orientation. For initial temperature above $200 \mathrm{~K}$, twins are observed to disappear in all cases while dislocations are not affected over the explored temperature range. The corresponding deformation anisotropy effects and initial conditions strongly affect subsequent bcc-hcp phase transition.

\section{Method and computational details}

Non Equilibrium Molecular Dynamics (NEMD) simulations were used to study deformation and bcchcp phase transition in iron crystal under planar ramp compression applied along the [001], [110] and [111] directions respectively. Samples with up to 28 millions atoms, $28.7 \mathrm{~nm} \times 28.7 \mathrm{~nm}(100$ $\left.a_{0} \times 100 a_{0}\right)$ cross section and length up to $402 \mathrm{~nm}\left(1400 a_{0}\right)$, either defect-free or containing 1.45 nm-radius microvoids ( $5 a_{0}$, with $a_{0}$ the lattice constant) regularly spaced by $28.7 \mathrm{~nm}\left(100 a_{0}\right)$ along the wave propagation direction were simulated. Furthermore, the initial temperature was varied from $50 \mathrm{~K}$ to $400 \mathrm{~K}$.

The simulations were realized using the Lammps molecular dynamics code[24] where interactions between atoms were modeled through the embedded atom model formalism[25, 26] expressed tangibly in the modified version of the Ackland potential[22]. This potential predicts the iron bcc to hcp phase transition at $13.75 \mathrm{GPa}$ under equilibrium hydrodynamic compression[22], in very good agreement with the experimental data[27, 28, 29]. It has also been successfully used in previous work to address plasticity and phase transition in [001]-oriented iron single crystal[23] and polycrystalline iron [30, 31] under shock compression.

In the present work, the ramp wave is initiated by driving an effective infinite-mass wall piston with an imposed velocity $v(t)$ along the z-direction, while periodic boundary conditions are applied in the transverse directions. The piston velocity increases linearly from 0 to Vmax within $30 \mathrm{ps,} \mathrm{with} \mathrm{Vmax}$ ranging from 800 to $1600 \mathrm{~m} / \mathrm{s}$. The corresponding strain rates are about $10^{8}-10^{9} \mathrm{~s}^{-1}$ comparable with that reported under picosecond-laser dynamic compression[32]. Local thermodynamic and mechanical 
variables such as longitudinal pressure $P_{z}$, deviatoric stress, temperature, etc. are evaluated within a spatial planar bin (of 3 lattice constant width) perpendicular to the wave propagation direction in the same manner as in our previous work[23]. Finally, local structural analysis is performed by adaptative Common Neighbor Analysis (CNA), Centrosymmetry and DXA, as implemented in the OVITO software[33].

\section{Results and Discussion}

\subsection{Load direction}

Depending on the direction of ramp compression, defect-free iron single crystal deformation process is found to be very different leading to a directional anisotropy in the bcc-hcp structural phase transformation features. Fig. 1 shows longitudinal pressure $P_{z}$ and shear stress profiles at two simulation times: 30 ps when the piston reached its maximum velocity and $40 \mathrm{ps}$. Corresponding atomic configurations are shown in Fig.2. For all propagation directions the sample first deforms elastically. The velocity of the elastic wave is found to depend on the loading direction with respect to crystal direction in consistency with previous MD simulations[34, 35]. In contrast with the [001] direction, in both [110] and [111] directions the elastic precursor is found to become steeper upon propagation thus leading to a sharp front at the foot of the compression wave. While no plasticity is clearly evident in the [111] direction before the structural phase transition, both [001] and [110] directions are found to exhibit plastic deformation.

In [001] direction, iron is found to yield via twinning at $P_{z}$ around $12 \mathrm{GPa}$ (see Fig. 3) with subsequent relaxation of the shear stress (see Fig. 2 and Fig. 1). Then, further compression is observed

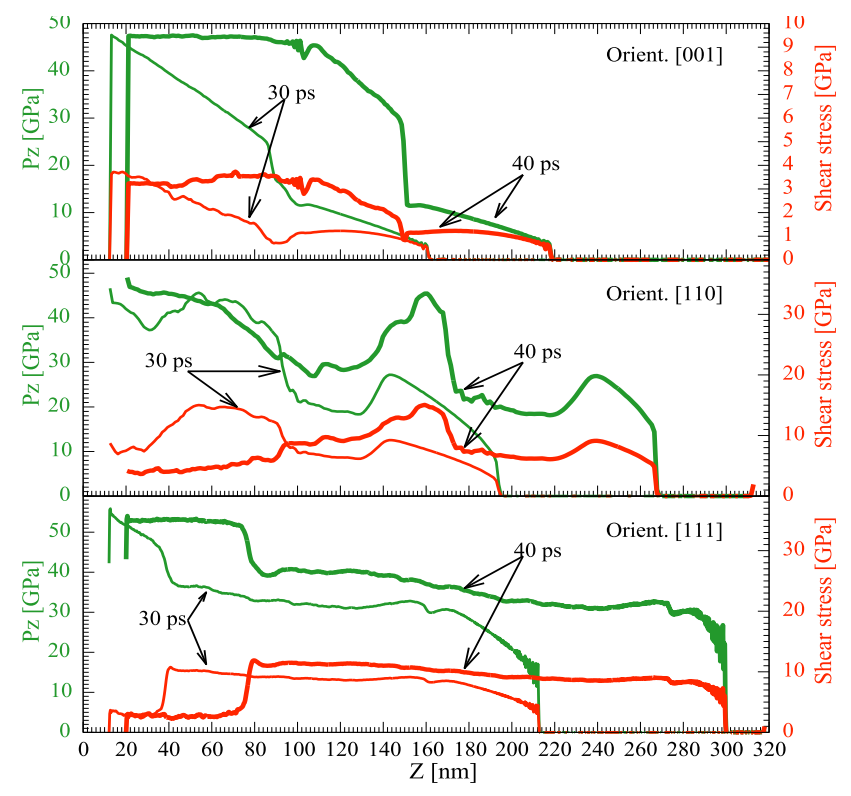

Figure 1: Wave profiles for [001] (top), [110] (middle) and [111] (bottom) crystallographic orientations of defect-free iron samples ramp compressed (with a piston maximum velocity of $800 \mathrm{~m} / \mathrm{s}$ ) at 30 picosecond (thin lines) when piston reached its maximum velocity and 10 ps later (thick lines). Green curves depict longitudinal pressure $P_{z}$ while red curves present shear stress. 

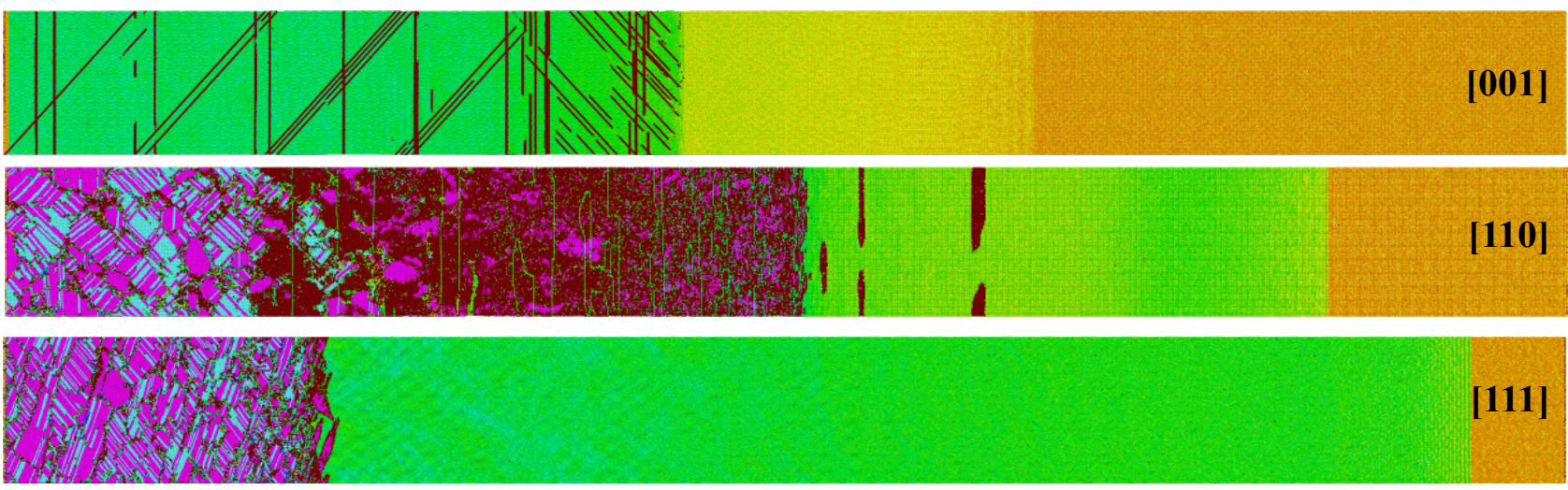

Figure 2: Atomic structure snapshots at 40 ps simulation time-step for [001] (top row), [110] (center row) and [111] (bottom row) directions in defect-free iron single crystal iron, ramp compressed to piston maximum velocity of $800 \mathrm{~m} . \mathrm{s}^{-1}$ (corresponding to maximum $P_{z}$ of about $50 \mathrm{GPa}$ ) within $30 \mathrm{ps}$. The non hcp and fcc atoms are colored according to $P_{z}$ (i.e. the z-component of the atomic stress $\sigma$ times the atomic volume $V_{a}$ ), except for atoms in twins, which are colored in brown. Violet and cyan are the hcp and fcc atoms respectively. Under compression along the [001] direction, twins accommodate plastic deformation above the elastic limit, with no phase transition. On the contrary, compression in the [111] direction remains elastic (no twins nor dislocations) up to the bcc-hcp phase transition. Finally, in the [110] direction, yield occurs via simultaneous twins formation and hcp nucleation, then the compressive wave splits into a twinning front followed by a metastable phase mixture where dislocations develop (see Fig. 4). Next, the bcc-hcp transition evolves to completion. The elastic wave is overdriven for both [110] and [111] directions.

to induce a hardening-like behavior, where the twins recede progressively without any dislocation activity, causing a new increase of the deviatoric stress. Such elastic stiffening of the bcc matrix

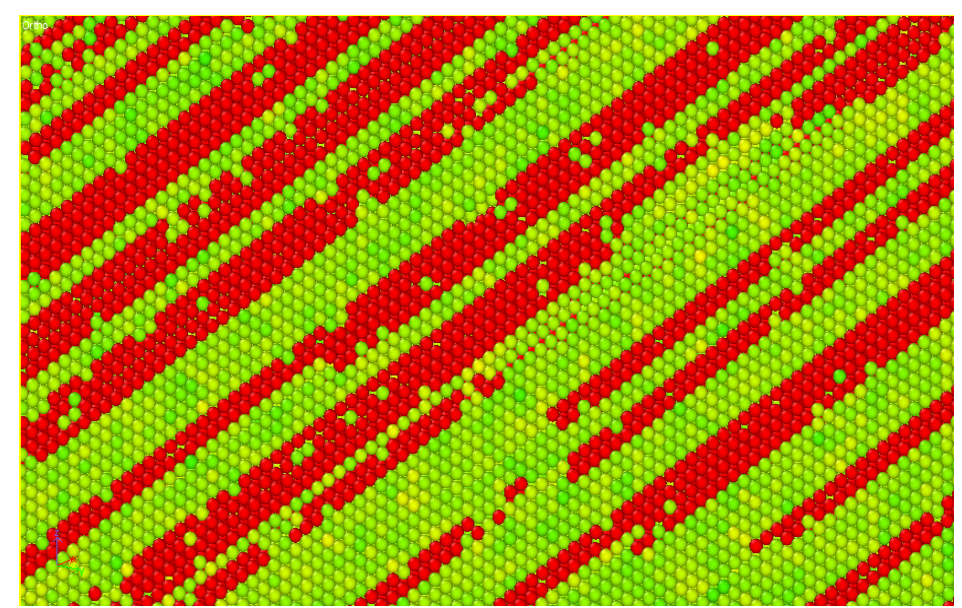

Figure 3: Example of atom configuration in the (11̄0) plane under ramp compression along [001]direction to a longitudinal pressure $\mathrm{Pz}$ of $19 \mathrm{GPa}$. Red lines are associated with twins. See ref [23] for these twins dynamics. 
inhibits the nucleation of the hcp phase, so that the onset of the phase transformation in the defectfree crystal is shifted to very high pressures. Thus, for the transition to be observed a pressure as high as $100 \mathrm{GPa}$ is required (see [23] for more details) which has been qualitatively explained by invoking phase nucleation and growth theory $[36,37]$ and the fact that a transformation from the elastic state would be expected to result in a significantly slower transformation kinetics [38]. In the present case, where maximum pressure remains below $50 \mathrm{GPa}$, no transition is observed (Fig. 2).

On the other hand, for [110] direction, the defect-free single crystal is found to remain perfectly elastic, with the shear stress linearly increasing, until the pressure reaches about $27 \mathrm{GPa}$ (Fig. 1). Then, yielding occurs via simultaneous nucleation of twins and hcp phase (only few fcc atoms are observed at this stage) inducing a rapid relaxation in the shear stress. Upon propagation, twinning and hcp homogeneous nucleation fronts split [3], (see Fig. 2). Like in the [001] direction, further compression induces a new increase of the shear stress indicative of some hardening-like effect. During this stage where the shear stress rises, the number of hcp atoms (and fcc) in the sample is observed
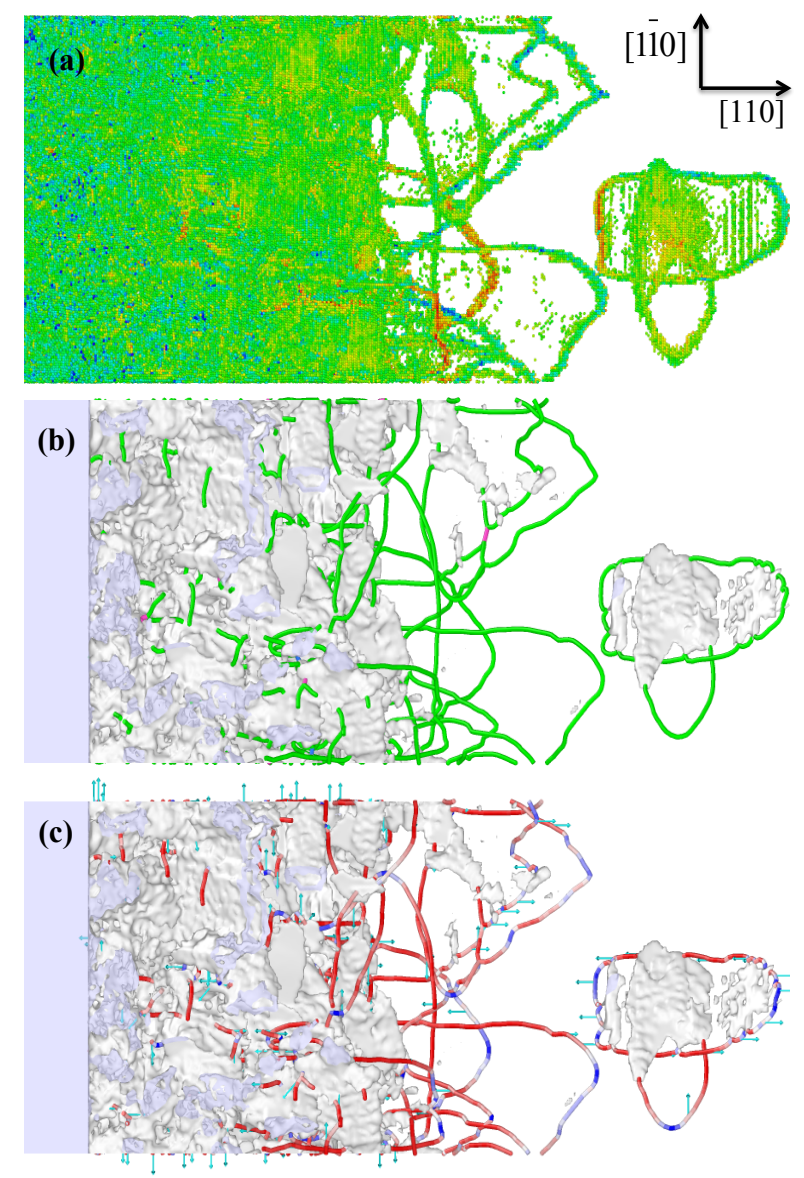

Figure 4: (a) Closer view of the mixed regime in a [110]-oriented defect-free crystal (Fig. 2, middle) showing particles with unknown coordination structure (by CNA analysis), identified by DXA analysis as dislocations loops. (b) Burgers vector type: dislocations are mainly $1 / 2<111>$ type (green) with only few $<100>$ type (violet, barely visible). (c) Dislocations local character: the red and blue lines are screw and edge dislocation types, respectively, while other colors represent mixed dislocation. Small arrows in pale blue are Burgers vectors (magnified by 10). The gray color corresponds to defect surfaces. 
to remain almost constant leading to a metastable mixed regime where coexist mainly bcc and hcp phases. The rapid growth stage of the bcc-hcp phase transition is thus delayed due to the stiffening effect. Meanwhile, when the pressure reaches a value around $40 \mathrm{GPa}$, a second yielding mechanism is triggered, namely homogeneous nucleation of dislocations followed by their rapid growth (see Fig. 4) producing a new shear stress relaxation (see Fig. 1). Here, the maximum density of dislocations is about $1.6 \times 10^{12} \mathrm{~cm}^{-2}$. The dislocations growth is accompanied by increasing number of fcc atoms while the number of hcp atoms decreases slightly until the shear stress attains 10 GPa. Only then, the hcp rapid growth regime is found to be triggered thus leading the structural phase transformation toward completion. The higher the piston velocity, the shorter structural phase transition time to completion, in agreement with experimental report in Cadmium sulfide (CdS) where plasticity was observed to play an important role in the structural phase transition nucleation and growth[38].

Thus, upon ramp loading along the [110] orientation, a double yielding is observed before the structural phase transition reaches its rapid growth regime. This behavior is consistent with the two-stage plasticity reported in other bcc single crystals dynamically compressed along their [110] direction, such as Mo in MD simulations combined with discrete to continuum method[39] or MgO in shock compression experiments[40].

The above complex deformation behavior contrasts with the response to compression along the [111] direction. Here, no sign of plastic deformation (twins or dislocations) is observed before the structural phase transformation, which is found to be the only way to relax shear stress in the defect-free crystal during the compression. This is consistent is with previous MD simulations of the iron single crystal response to both shock compression[34, 35] and non-equilibrium uniaxial deformation [41] along [111] direction. When the longitudinal pressure $P_{z}$ reaches about $30 \mathrm{GPa}$ some atoms are observed to start moving transversally to the compression direction, leading to a plateau in the pressure profile (see 1). As a result, the shear stress increase also slows down. Then, the transition occurs when the pressure $P_{z}$ reaches $35 \mathrm{GPa}$ with sharp drop in the shear stress down to around $4 \mathrm{GPa}$. The duration of this regime which could be identified with transition incubation period was observed to depend on the piston maximum velocity, i.e the maximum pressure[38]. The higher the piston maximum velocity the shorter the plateau duration. So, neither a hardening-like effect nor a mixed regime are observed in contrast to the other investigated directions.

Significant fractions of fcc atoms are found by the CNA analysis (see Fig. 2) in both [110] (hcp/fcc ratio $\approx 2$ ) and [111] (hcp/fcc ratio $\approx 2.7)$. Although such hcp-fcc mixture was already predicted in MD simulations based on the same iron potential[30], as well as by other potentials[34, 35], it has not been experimentally evidenced yet, e.g.[42]. Still, detecting such mixed hcp-fcc phases would require accurate probing of the local crystal structure at the atomic level over ultra-short time scales, which remains very challenging, especially since it was inferred from MD data, that the fcc phase found by CNA analysis does not result in any prominent feature in the X-ray diffraction pattern[30].

\subsection{Lattice defects}

The presence of initial defects in a material is known to be an important factor that determines its mechanical response[43]. Real materials contain such defects as processing and handling may intrinsically introduce inclusions, voids or imperfections even in single crystal[44]. Following[45], in order to investigate the influence of such defective initial conditions on both plasticity and phase transition microvoids of $5 a_{0}$-radius (with $a_{0}$ the lattice constant) were introduced every $100 a_{0}$ along 


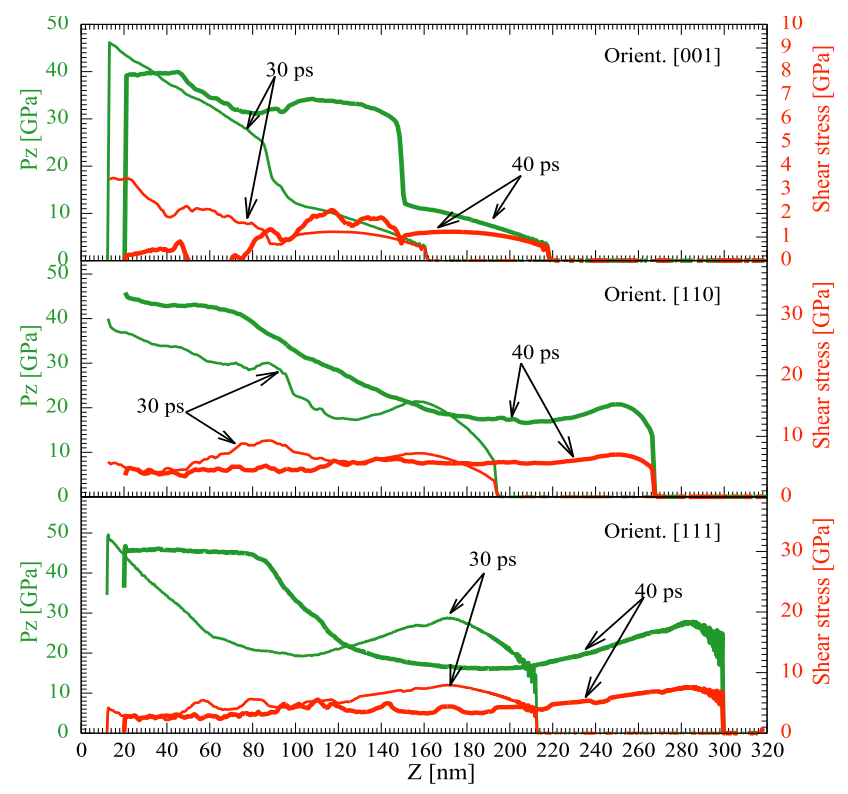

Figure 5: Wave profiles in [001] (top), [110] (middle) and [111] (bottom) -oriented iron single crystals containing initial defects in the form of microvoids (see text for details), at 30 ps when piston reached its maximum velocity of $800 \mathrm{~m} / \mathrm{s}$ and $10 \mathrm{ps}$ later. Green curves depict longitudinal pressure $P_{z}$ while red curves represent shear stress

the loading direction. Figures 5 and 6 show the wave profiles and the atomic configurations snapshots respectively at simulation time step of $40 \mathrm{ps}$. In [001]-oriented crystal, twinning is found to be the predominant deformation mechanism above a yielding pressure value of about $P_{z}=12 \mathrm{GPa}$, almost the same as in the defect-free case. It is followed by a stage of elastic stiffening, where the shear stress

\section{5}

$\mathbf{P}_{\mathrm{z}} \mathbf{V}_{\mathrm{a}}[\mathrm{eV}]$
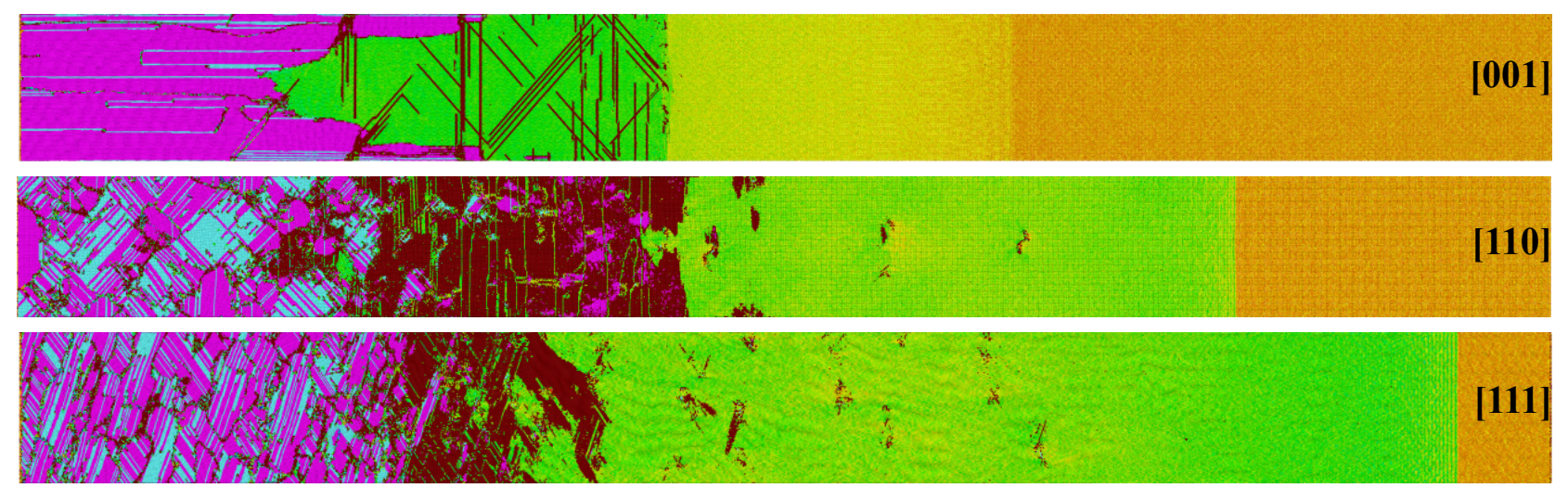

Figure 6: Atomic structure snapshots at 40 ps for [001] (top row), [110] (center row) and [111] (bottom row) directions in defective iron single crystal ramp compressed to piston maximum velocity of 800 $m . s^{-1}$ (corresponding to maximum $P_{z}$ of about $50 \mathrm{GPa}$ ) within $30 \mathrm{ps.} \mathrm{Atoms} \mathrm{color} \mathrm{codes} \mathrm{are} \mathrm{the}$ same as in Fig. 2. Deformation is characterized by dislocations emission from micro-voids (see Fig. 7) before the bcc to hcp phase transition. Plasticity under compression along the [001] direction remains dominated by twins. 
increases again.

Unlike in previous MD simulations of defective iron single crystal with Voter-Chen interatomic potential[45, 46], the emission of dislocations from microvoids upon further compression is observed (around a local $P_{z}$ of $17 \mathrm{GPa}$ ). This behavior is consistent with that reported for other bcc materials such as tantalum[47]. However the growth of these dislocations is found to be very slow. Even longer lasting simulation over 200 ps showed neither accelerated growth nor homogeneous nucleation of dislocations in the [001]-oriented crystal. Nevertheless, in the presence of microvoids and subsequent emitted dislocations, no shifting of the phase transition to a high pressure was observed, which is consistent with the theory of heterogeneous nucleation[36, 37, 48]. In the context of this theory, the presence of defects allows the nuclei of new phase [49] to develop in the parent phase near defects where the work of nucleation is much reduced[36]. So, in contrast with the defect-free crystal, despite some elastic stiffening of the bcc matrix, the transition is made locally possible by an important reduction in its free energy barrier. Thus, the structural phase transition is now observed to start near voids when the local $P_{z}$ reaches $\sim 25 \mathrm{GPa}$, which is very low compared to the $100 \mathrm{GPa}$ in the case of defect-free crystal[23]. Then, it grows for about $20 \mathrm{ps}$ leading to a kind of plateau in the pressure profile (at the top of the plastic wave) at around $30 \mathrm{GPa}$ where shear stress starts to relax (see Fig. 5). Upon completion of the structural phase transformation, the shear stress is completely relaxed and even over-relaxed[34, 20, 41]. This illustrates the importance of defects in the development of martensitic phase transformation.

Upon compression along the [110] direction with defective initial conditions, dislocations loops are first observed to nucleate from microvoids when the $P_{z}$ reaches locally about $20 \mathrm{GPa}$. At this stage, neither twinning nor hcp nucleation occurred in the sample. Then the dislocation loops expand through the sample by stretching (see Fig. 7), occasioning a first shear stress relaxation (see Fig. 5 at $30 \mathrm{ps}$ ). Here, the pressure at the top of the elastic wave associated with the yielding pressure, after a slight decrease during wave propagation, is found to be established around 20 GPa, i.e at the local pressure value of dislocations emission. This value is lower than in the defect-free case $(\approx 27 \mathrm{GPa})$ in

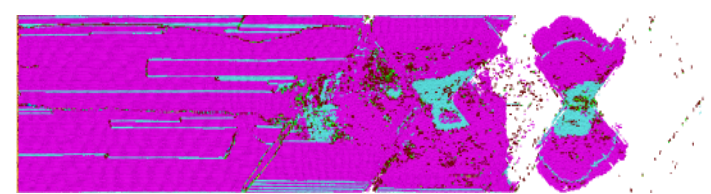

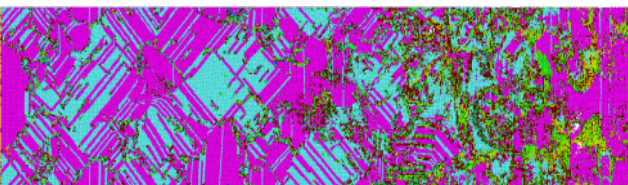

(1)

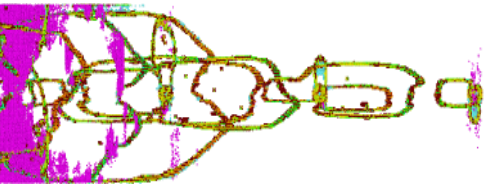

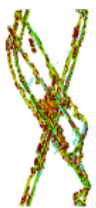

는

(1)

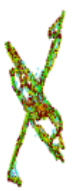

- $[001]$ e

- $[110]$ ค

[111]。

Figure 7: Non bcc atoms structure referring Fig. 6 with the top, center and bottom images corresponding to [001], [110] and [111] directions respectively. Color codes are the same as in Fig. 2. Dislocations loops (corresponding here to atoms with non identified structure by cna analysis) are clearly evidenced around micro-voids. 
agreement with the fact that without initial defect, crystal strength is over-predicted[43]. Upon further compression, hcp phase is found to nucleate either on the dislocation loop or from the voids. While twinning generally accompanyies the hcp phase nucleation, twinning alone is also found to develop directly on the dislocations loops as it was reported in refs[50, 43]. Next, the deformation process becomes similar to that of the defect-free crystal with the formation of a mixed regime and hardening like behavior until homogeneous nucleation of dislocations at pressure $P_{z}$ of about $38 \mathrm{GPa}$. Then, the shear stress relaxes again (see Fig. 5, profiles at $30 \mathrm{ps)}$ ). Later when the sample is homogeneously filled by dislocations, the shear stress decreases upon further compression with no double yielding (see Fig. 5, profiles at $40 \mathrm{ps}$ ). The pressure at the front of the mixed regime that we consider as the onset pressure for the phase transition is around $22 \mathrm{GPa}$, which is almost the same as the yielding pressure.

Finally, in the [111] oriented crystal with defective initial configuration, dislocations emission starts above $20 \mathrm{GPa}$, which is comparable to that in the [110] direction. Then dislocations grow within less than 10 ps. An important pressure relaxation precedes the structural phase transition instead of pressure stagnation. Although twinning is found to accompany the dislocation development, no massive homogeneous nucleation of hcp phase is observed (see Fig. 7). In this case, the bcc-hcp phase transition is found to start at pressure $P_{z}$ of about $33 \mathrm{GPa}$, then grow whithin about $10 \mathrm{ps}$ kinetics time scale up to completion around $40 \mathrm{GPa}$.

So, while with defective initial conditions the deformation remains mainly dominated by twinning when compression is applied along the [001] direction, dislocations mediated deformation is the favored way of shear stress relaxation for the crystal loaded along both [110] and [111] directions.

Fig. 8 shows the nucleation and growth of dislocations from microvoids for compression along the [110] direction. Mainly $1 / 2<111>$ Burgers vector type dislocations are nucleated in the form of loops. Each loop consists of two screw dislocations (with opposite Burgers vectors) moving apart, separated by a leading curved non-screw line (mixed plus edge types) that propagates away from the void, in consistence with experimental observation in pure iron under in situ straining experiments [51, 52]. When, the leading non-screw dislocation part meets another dislocations loop (or a microvoid), it slows down.

The interactions between different loops lead to dislocations multiplication that establishes a dense network in the sample (see Fig.7 (b) and (c)). Here, the maximum dislocation density is found to be

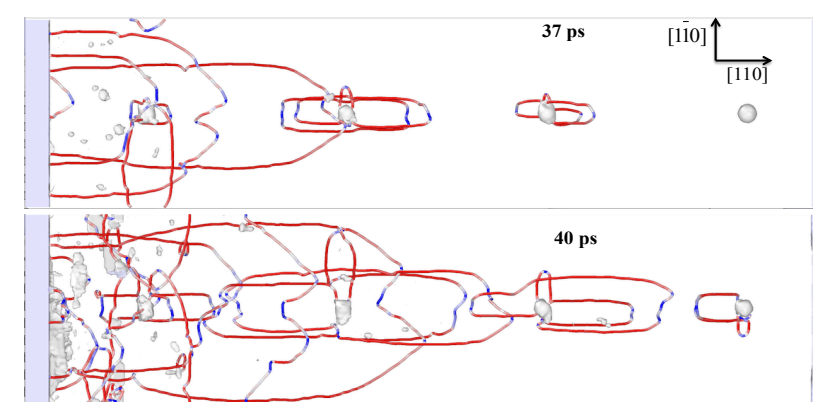

Figure 8: Nucleation and growth of dislocation loops between $37 \mathrm{ps}$ and $40 \mathrm{ps}$ in a crystal containing initial defects, ramp-compressed along its [110] direction. Burgers vectors are almost all of $1 / 2<111>$ type. Loops consist of two screw dislocations with opposite Burgers vectors (in red) separated by edge dislocations (in blue), which propagate through the sample. 
at around $1.4 \times 10^{12} \mathrm{~cm}^{-2}$ almost identic to the defect-free case. Furthermore, the, screw dislocations are found to move with average velocities around $397 \mathrm{~m} / \mathrm{s}$ while the leading edge component velocity is found to be approximately $2600 \mathrm{~m} / \mathrm{s}$. Thus, the edge component moves faster than the screw one, which agrees with experimental observations[51, 52]. A similar behavior is observed for compression along [111]-direction. Here, dislocation density is about $4.3 \times 10^{12} \mathrm{~cm}^{-2}$ while the screw and edge component velocities are found to be $585 \mathrm{~m} / \mathrm{s}$ and $2400 \mathrm{~m} / \mathrm{s}$, respectively. In contrast, when compression is applied along the [001] direction, dislocations are mostly of edge type (again with essentially $1 / 2<111>$ Burgers vector), and their velocity is too slow to be determined (see Fig. 7(a)).

As the ramp compression is accompanied by high shear stress for both [110] and [111] directions (see Fig. 5), dislocations must flow faster in order to allow the material to deform and relieve the high shear stress on the rapid time scale of the compression[7]. Thus the dislocation velocities found here are higher than that found under quasi-static loading[51]. However, these velocities are qualitatively consistent with previous MD simulations in iron[50] and other bcc material such as tantalum in both $\operatorname{MD}[53,54]$ and stochastic [55] simulations.

\subsection{Initial temperature effect}

In order to investigate the effects of initial temperature on the deformation and structural phase transition, simulations (with or without defects) were done where the lattice equilibration temperature was varied from 50 to $400 \mathrm{~K}$ before ramp compression. Upon elevation of the initial temperature from $50 \mathrm{~K}$ to $300 \mathrm{~K}$, the dislocations nucleation and growth are observed to remain similar, i.e. emission of mainly $1 / 2<111>$ dislocation loops composed with two screw dislocation lines connected by a curved non-screw leading part for both [110] and [111] directions. However, the dislocation densities and velocities are found to increase slightly. Indeed, for compression along [110]-direction ([111]direction) with an initial temperature of $300 \mathrm{~K}$, the dislocation density is found to be $2.7 \times 10^{12} \mathrm{~cm}^{-2}$ $\left(5.3 \times 10^{12} \mathrm{~cm}^{-2}\right)$. On the other hand, the screw and edge velocities for [110]-direction ([111]-direction) are $506 \mathrm{~m} / \mathrm{s}(820 \mathrm{~m} / \mathrm{s})$ and $3000 \mathrm{~m} / \mathrm{s}(2500 \mathrm{~m} / \mathrm{s})$, respectively. Such influence of temperature on the velocity is qualitatively consistent with trends reported in the literature[55, 50, 56]. When compression is applied along the [001] direction, dislocations are mostly of edge type, and their velocity is still too slow to be determined. A more complete and detailed analysis of the dislocation behavior will be presented in a forthcoming paper.

In contrary, the occurrence of deformation twinning is found to be strongly temperature-dependent. In fact, no twinning is observed during the compression in either defect-free or defective crystal when initial temperature equals or exceeds $200 \mathrm{~K}$ (see Fig. 9). This indicates that the deformation twinning occurring in our previous simulations is mostly a low-temperature effect of iron single crystal in consistency with the well known fact that low temperature favors twinning and with the existence of twinning temperature limit[57,58]. Thus, on varying the initial temperature both plastic deformation and structural phase transformation characteristics are found to remain almost identical to $50 \mathrm{~K}$ simulations for defect-free [111]-oriented already twin-free at low temperature and defective both [110] and [111]-oriented crystals where the low temperature deformation was mainly dominated by dislocations. This behavior is coherent with previous MD results reported in iron using Voter-Chen potential where increasing the initial temperature from 50 to $300 \mathrm{~K}$ has no significant influence[34]. On the other hand, along the [110], by increasing the temperature above $200 \mathrm{~K}$, the first stage of the deformation is found to become similar to that of the [111] direction in defect-free crystal at $50 \mathrm{~K}$, i.e elastic deformation 

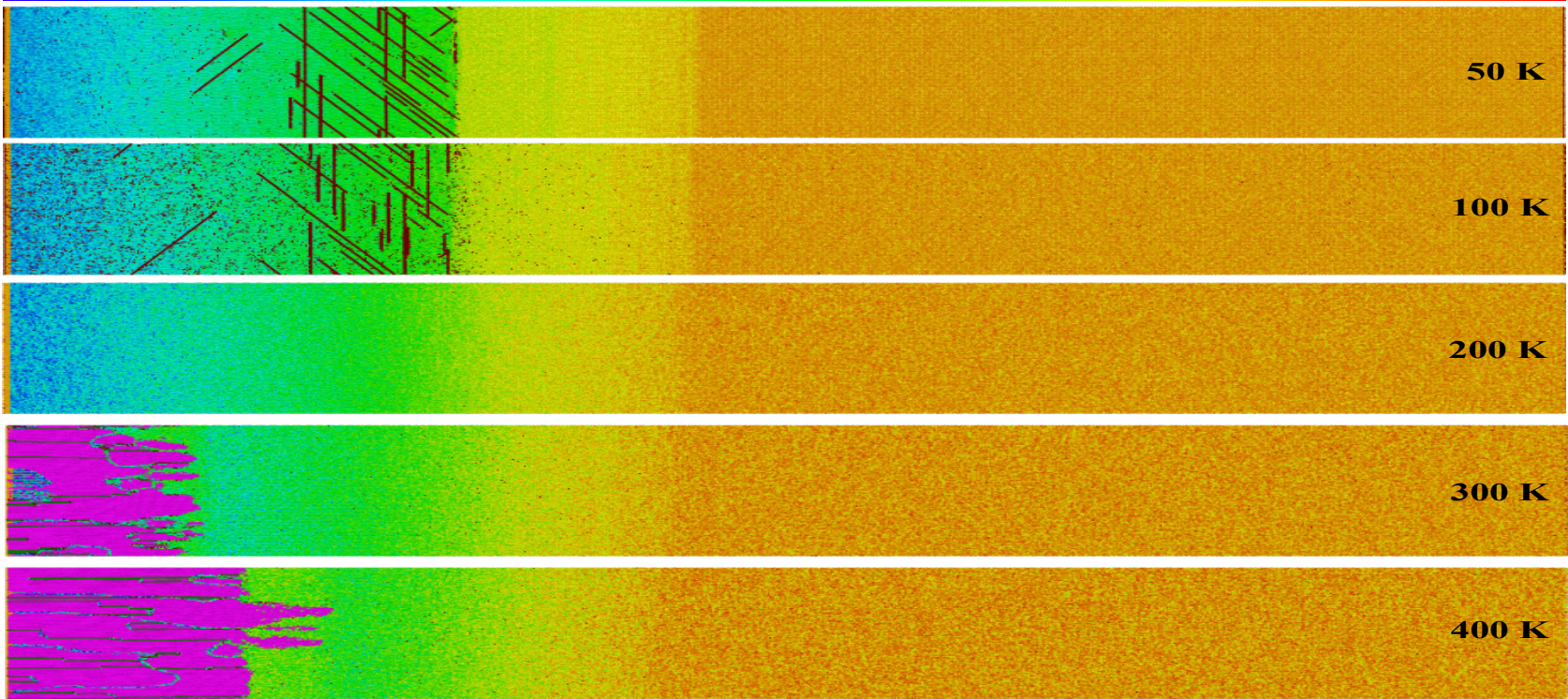

Figure 9: Atomic structure snapshots at 20 ps simulation time-step at different equilibration- temperatures along [001] direction for defect-free iron single crystal iron, ramp compressed during 30 ps to piston maximum velocity of $1600 \mathrm{~m} . \mathrm{s}^{-1}$ (corresponding to maximum $P_{z}$ of about $110 \mathrm{GPa}$ ). The snapshots from top to bottom correspond to 50, 100, 200, 300 and 400K initial temperature respectively. Atoms color codes are the same as in the Fig. 2. See text for details.

followed by pressure stagnation before the structural phase transformation. Then, the deformation proceeds by a mixed regime in which dislocations develop as in low temperature regime. So, no double yielding is observed.

Besides, along [001] direction (with or without microvoids) where twinning was the main plastic response at low temperature, the crystal response evolves from elastic-plastic to perfect elastic when the initial temperature is $400 \mathrm{~K}$. Here, the shear stress increases linearly with compression until the structural phase transformation proceeds in an elastic state (Fig. 10). The amplitude of atomic thermal fluctuations is the only factor that favors the structural phase transition; the transition onset pressure is observed to decrease from about $100 \mathrm{GPa}$ at $50 \mathrm{~K}$ to about $40 \mathrm{GPa}$ at $400 \mathrm{~K}$. This is consistent with

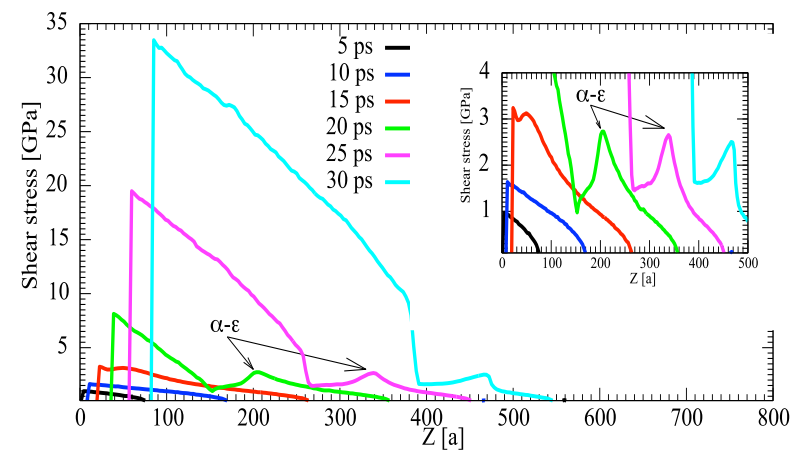

Figure 10: Profiles of shear stress for [001]-oriented single iron sample initially equilibrated at temperature of 400K. See also Fig. 9. 
the martensitic character of this transition in agreement with both negative slope of the bcc-hcp phase boundary in the pressure-temperature diagram and previous experimental works on ramp compression where the transition velocity was observed to decrease on increasing the target initial temperature[59]. These results show that the temperature is a key factor for understanding deformation and structural phase transition in single crystal iron. Furthermore, they also give insight into the lack of plasticity when shock wave propagates along [001] direction in iron single crystal under laser compression where initial temperature is usually about $300 \mathrm{~K}$ (ambient conditions), or higher (in cases of preheating due to laser-plasma interaction) $[44,15 ?]$.

\section{Summary and Conclusions}

In summary, the effects of orientation, defects and initial temperature on the response of iron single crystals to ramp compression have been investigated using the modified version of the Ackland potential[22]. In contrast with results obtained with the Voter Chen potential[34, 41], the elastic-plastic deformation behavior is found to strongly depend on crystal orientation, with major consequences on both the onset pressure and the kinetics of the bcc-hcp phase transition (Fig. 11). As reported previously[23], ramp compression along the [001] direction from a $50 \mathrm{~K}$ initial temperature produces twin-mediated yielding (at $\mathrm{Pz}$ around $12 \mathrm{GPa}$ ) followed by elastic hardening which inhibits the structural phase transition (up to very high pressures on the order of $100 \mathrm{GPa}$ ). However, compression along other crystal orientations leads to very different responses. While perfect crystals compressed in the [111] direction remain basically elastic up to the onset of the phase transition (at Pz around $35 \mathrm{GPa}$ ), those compressed in their [110] direction exhibit a complex behavior, with simultaneous twinning and massive nucleation of hcp phase (at about $27 \mathrm{GPa}$ ) creating a mixed-phase, metastable regime where dislocations develop (above about $40 \mathrm{GPa}$ ). The overall plasticity deformation behavior, i.e twinning along [001] direction and twinning followed by dislocations along [110] direction is consistent with features previously observed in defect-free bcc material such as tantalum[60, 43]. They differ from those obtained with the modified analytic embedded-atom-model potential for which plasticity, fully dislocation-mediated, is only found under compression in the [110] direction above $17 \mathrm{GPa}[35]$.

The strong directional dependence of the bcc-hcp phase transition reported above is consistent with the theory of phase nucleation and growth[36, 37, 48]. Indeed, the thermodynamic driving force for the phase transformation to occur results from the negative bulk (chemical) free energy difference between the parent and product phases. However, the formation of nucleus of the product phase induces positive interface energy, which opposes to the transformation. Besides, in the solid state, as the product and parent phases have different crystalline structures and specific volumes, the transformation is accompanied by dimensional changes. Such changes in volume and shape cannot occur freely because of the rigidity of the surrounding matrix, and strain energy is induced, which tends to inhibit the growth of the nucleus[37]. Thus, a different plastic behavior along different crystallographic orientations would be expected to affect the onset of the phase transition, in consistence with our simulations and experimental observations in other shock-compressed materials such as potassium chloride[61] and cadmium sulfide[38].

However, this observation contrasts with plate impact experiments on iron single crystal[62] where, although elastic-plastic deformation was found to be anisotropic, the same transition onset pressure was measured for different directions of shock application. Here, several possible origins have been 


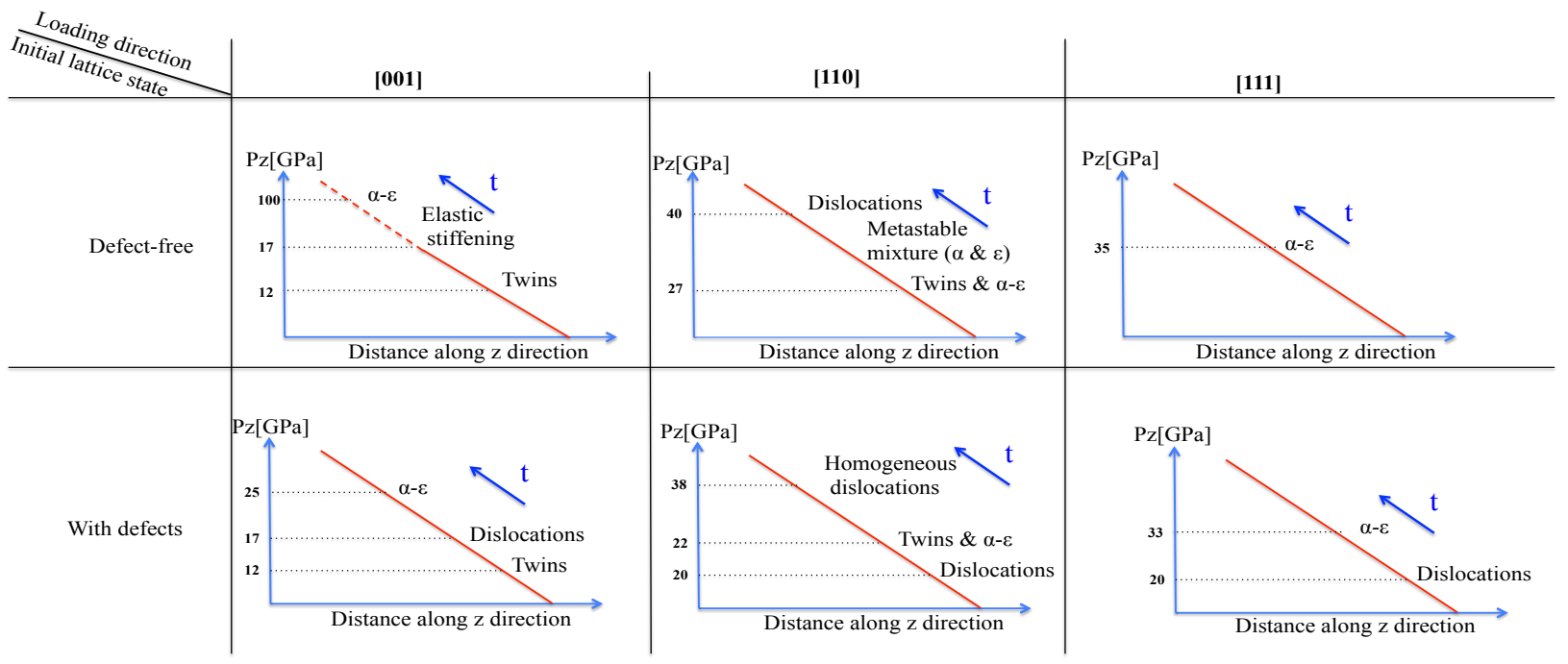

Figure 11: Summary of the processes (with their onset pressure $P_{z}$ ) governing the response to ramp compression along the z-direction. The arrows present the time sequence in which those processes successively occur. The initial temperature of the crystal is $50 \mathrm{~K}$. If this temperature is increased above about $200 \mathrm{~K}$, twinning disappears in all cases.

invoked to explain the discrepancy between the experiment and shock MD simulations among which different time scales (four magnitude order of difference) and different shear stress state during the compression[62]. Indeed, it is suggested that during the experiment the phase transition would happen under nearly hydrostatic conditions, after full completion of the plastic processes and subsequent yielding, very different from the transient conditions and high shear stresses involved in our simulations (see Fig.1 and Fig.5)

Besides, this observation is also consistent with other experiments involving laser driven and magnetic driven ramp compression of polycrystalline iron[14], as well as shock compression of other materials such as CdS, where the transformation kinetics was found to depend significantly on the crystallographic orientation[38]. Regarding high rate effects considered herein, under ramp compression involving strain rates above $10^{6} \mathrm{~s}^{-1}$, a transition is expected from thermal activation to phonon drag plasticity regime, where the growth of the new-phase nuclei within the parent matrix is limited by phonon drag mediated dislocation velocity[14].

The introduction of initial defects in the form of microvoids favors plastic deformation, which is then observed in all three loading directions, above a yielding pressure of $12 \mathrm{GPa}$ ([001] direction) or $20 \mathrm{GPa}$ ([110] and [111] directions). Such anisotropic elastic limit, in violation of the Schmid law, is characteristic of bcc materials at low temperature[63, 64], and originates from the behavior of screw dislocations. Indeed, in the presence of defects, plasticity is still dominated by twinning in the [001] case, due to a very slow dislocation kinetics in this loading direction, while it is mainly mediated by (screw) dislocations activity in the other two directions. Like in defect-free crystals, directional dependence is also found for the onset pressure of the structural phase transition and its kinetics (Fig. $11)$.

The values of this onset pressure (25, 22 and $33 \mathrm{GPa}$ for [001], [110] and [111] directions, respectively) are lower than their counterparts in the defect-free crystal, which is consistent with the theory of heterogeneous nucleation. They are logically higher than the $\sim 13$ GPa equilibrium value due to 
the very short compression times considered here, while they are in good quantitative agreement with the $25 \mathrm{GPa}$ found under picosecond laser compression[32] and with the 11-38 GPa range (depending on the strain rate) found under nanosecond laser ramp compression[14, 15, 16], as well as with the 23 GPa predicted by recent MD simulations of shock waves in polycrystalline iron [30].

\section{Acknowledgments}

Computations were performed on the supercomputer facilities of the Mésocentre de calcul de Poitou Charentes (France).

\section{Data availability}

The raw/processed data required to reproduce these findings cannot be shared at this time as the data also forms part of an ongoing study.

\section{References}

[1] M. A. Meyers. Dynamic behavior of materials. John Wiley and Sons, New York, 1994. pp:1-21.

[2] B. A. Remington, R. E. Rudd, and J. S. Wark. From microjoules to megajoules and kilobars to gigabars: Probing matter at extreme states of deformation. Physics of Plasmas, 22(9):090501, 2015.

[3] G. E. Duvall and R. A. Graham. Phase transitions under shock-wave loading. Rev. Mod. Phys., 49:523-579, 1977.

[4] B. A. Remington, G. Bazan, J. Belak, E. Bringa, J. D. Colvin, M. J. Edwards, S. G. Glendinning, D. H. Kalantar, M. Kumar, B. F. Lasinski, K. T. Lorenz, J. M. McNaney, S. M. Pollaine, D. Rowley, J. S. Stölken, S. V. Weber, W. G. Wolfer, M. Caturla, D. S. Ivanov, L. V. Zhigilei, B. Kad, M. A. Meyers, M. Schneider, D. D. Meyerhofer, B. Yaakobi, and J. S. Wark. Materials science under extreme conditions of pressure and strain rate. Metallurgical and Materials Transactions A, 2004.

[5] B. A. Remington, P. Allen, E. M. Bringa, J. Hawreliak, D. Ho, K. T. Lorenz, H. Lorenzana, J. M. McNaney, M. A. Meyers, S. W. Pollaine, K. Rosolankova, B. Sadik, M. S. Schneider, D. Swift, J. Wark, and B. Yaakobi. Material dynamics under extreme conditions of pressure and strain rate. Materials Science and Technology, 22(4):474-488, 2006.

[6] H. E. Lorenzana, J. F. Belak, K. S. Bradley, E. M. Bringa, K. S. Budil, J. U. Cazamias, B. ElDasher, J. A. Hawreliak, J. Hessler, K. Kadau, D. H. Kalantar, J. M. McNaney, D. Milathianaki, K. Rosolankova, D. C. Swift, M. Taravillo, T. W. Van Buuren, J. S. Wark, and T. Diaz de la Rubia. Shocked materials at the intersection of experiment and simulation. Sci. Model Simul., 15:159-186, 2008.

[7] R. E. Rudd, T. C. Germann, B. A. Remington, and J. S. Wark. Metal deformation and phase transitions at extremely high strain rates. MRS Bulletin, 35(12):999-1006, 2010. 
[8] M. A. Meyers and L. E. Murr. Defect generation in shockwave deformation. Shock Waves and High-Strain-Rate Phenomenena in Metals, page 487, 1981.

[9] Y. B. Ya. B. Zel'dovich and Y. P. Raizer. Physics of Shock Waves and High-Temperature Hydrodynamic Phenomena. 2002. pp: 685-784.

[10] R. F. Smith, J. H. Eggert, R. E. Rudd, D. C. Swift, and C. A. Bolme et al. High strain-rate plastic flow in Al and Fe. J. Appl. Phys., 110(12):123515, 2011.

[11] J. R. Asay. The use of shock-structure methods for evaluating high-pressure material properties. International Journal of Impact Engineering, 20(1-5):27-61, 1997.

[12] J. Edwards, K. T. Lorenz, B. A. Remington, S. Pollaine, J. Colvin, D. Braun, B. F. Lasinski, D. Reisman, J. M. McNaney, J. A. Greenough, R. Wallace, H. Louis, and D. Kalantar. Laserdriven plasma loader for shockless compression and acceleration of samples in the solid state. Phys. Rev. Lett., 92:075002, 2004.

[13] N. Amadou, E. Brambrink, A. Benuzzi-Mounaix, G. Huser, F. Guyot, S. Mazevet, G. Morard, T. de Resseguier, T. Vinci, K. Myanishi, N. Ozaki, R. Kodama, T. Boehly, O. Henry, D. Raffestin, and M. Koenig. Direct laser-driven ramp compression studies of iron: A first step toward the reproduction of planetary core conditions. High Energy Density Physics, 9(2):243 - 246, 2013.

[14] R. F. Smith, J. H. Eggert, D. C. Swift, J. Wang, T. S. Duffy, D. G. Braun, R. E. Rudd, D. B. Reisman, J.-P. Davis, M. D. Knudson, and G. W. Collins. Time-dependence of the alpha to epsilon phase transformation in iron. J. Appl. Phys., 114(22):223507, 2013.

[15] N. Amadou, T. de Resseguier, E. Brambrink, T. Vinci, A. Benuzzi-Mounaix, G. Huser, G. Morard, F. Guyot, K. Miyanishi, N. Ozaki, R. Kodama, and M. Koenig. Kinetics of the iron $\alpha-\varepsilon$ phase transition at high-strain rates: Experiment and model. Phys. Rev. B, 93:214108, 2016.

[16] N. Amadou, E. Brambrink, T. de Rességuier, A. O. Manga, A. Aboubacar, B. Borm, and A. Molineri. Laser-driven ramp compression to investigate and model dynamic response of iron at high strain rates. Metals, 6(12), 2016.

[17] A. Vattré and C. Denoual. Polymorphism of iron at high pressure: A 3d phase-field model for displacive transitions with finite elastoplastic deformations. Journal of the Mechanics and Physics of Solids, 92:1 - 27, 2016.

[18] B. L. Holian and P. S. Lomdahl. Plasticity induced by shock waves in nonequilibrium moleculardynamics simulations. Science, 280(5372):2085-2088, 1998.

[19] T. C. Germann, B. L. Holian, P. S. Lomdahl, and R. Ravelo. Orientation dependence in molecular dynamics simulations of shocked single crystals. Phys. Rev. Lett., 84:5351-5354, 2000.

[20] K. Kadau, T. C. Germann, P. S. Lomdahl, and B. L. Holian. Microscopic view of structural phase transitions induced by shock waves. Science, 296(5573):1681-1684, 2002.

[21] E. M. Bringa, K. Rosolankova, R. E. Rudd, B. A. Remington, J. S. Wark, M. Duchaineau, D. H. Kalantar, J. Hawreliak, and J. Belak. Shock deformation of face-centred-cubic metals on subnanosecond timescales. Nature Materials, 5:805-809, 2006. 
[22] N. Gunkelmann, E. M. Bringa, K. Kang, G. J. Ackland, C. J. Ruestes, and H. M. Urbassek. Polycrystalline iron under compression: Plasticity and phase transitions. Phys. Rev. B, 86:144111, 2012.

[23] N. Amadou, T. de Resseguier, A. Dragon, and E. Brambrink. Coupling between plasticity and phase transition in shock- and ramp-compressed single-crystal iron. Phys. Rev. B, 98:024104, 2018.

[24] S. Plimpton. Fast parallel algorithms for short-range molecular dynamics. J. Comp. Phys., 117:119, 1995.

[25] M. S. Daw and M. I. Baskes. Semiempirical, quantum mechanical calculation of hydrogen embrittlement in metals. Phys. Rev. Lett., 50:1285-1288, 1983.

[26] S. M. Foiles, M. I. Baskes, and M. S. Daw. Embedded-atom-method functions for the fcc metals $\mathrm{Cu}, \mathrm{Ag}, \mathrm{Au}, \mathrm{Ni}, \mathrm{Pd}, \mathrm{Pt}$, and their alloys. Phys. Rev. B, 33:7983-7991, 1986.

[27] D. Bancroft, E. L. Peterson, and S. Minshall. Polymorphism of iron at high pressure. Journal of Applied Physics, 27(3):291-298, 1956.

[28] L. M. Barker and R. E. Hollenbach. Shock wave study of the $\alpha-\varepsilon$ transition in iron. J. Appl. Phys., 45(11):4872-4887, 1974.

[29] J. C. Boettger and D. C. Wallace. Metastability and dynamics of the shock-induced phase transition in iron. Phys. Rev. B, 55:2840-2849, 1997.

[30] N. Gunkelmann, E. M. Bringa, D. R. Tramontina, C. J. Ruestes, M. J. Suggit, A. Higginbotham, J. S. Wark, and H. M. Urbassek1. Shock waves in polycrystalline iron: Plasticity and phase transitions. Phys. Rev. B, 89:140102, 2014.

[31] N. Gunkelmann, D. R. Tramontina, E. M. Bringa, and H. M. Urbassek. Interplay of plasticity and phase transformation in shock wave propagation in nanocrystalline iron. New J. Phys., 16(9):093032, 2014.

[32] J. C. Crowhurst, B. W. Reed, M. R. Armstrong, H. B. Radousky, J. A. Carter, D. C. Swift, J. M. Zaug, R. W. Minich, N. E. Teslich, and M. Kumar. The $\alpha-\varepsilon$ phase transition in iron at strain rates up to $10^{9} s^{-1}$. J. Appl. Phys., 115(11):113506, 2014.

[33] A. Stukowski. Visualization and analysis of atomistic simulation data with OVITO-the open visualization tool. Modelling and Simulation in Materials Science and Engineering, 18:015012, 2009 .

[34] K. Kadau, T. C. Germann, P. S. Lomdahl, and B. L. Holian. Atomistic simulations of shockinduced transformations and their orientation dependence in bcc Fe single crystals. Phys. Rev. B, 72:064120, 2005.

[35] K. Wang, S. Xiao, H. Deng, W. Zhu, and W. Hu. An atomic study on the shock-induced plasticity and phase transition for iron-based single crystals. Int. J. Plasticity, 59:180 - 198, 2014.

[36] J.W. Christian. The Theory of Transformations in Metals and Alloys. Elsevier, 2002. pp: 422-479. 
[37] R. E. Smallman and A. H. W. Ngan. Modern Physical Metallurgy. Elsevier, 2014.

[38] M. D. Knudson and Y. M. Gupta. Transformation kinetics for the shock wave induced phase transition in cadmium sulfide crystals. J. Appl. Phys., 91(12):9561-9571, 2002.

[39] R. Kositski, D. Steinberger, S. Sandfeld, and D. Mordehai. Shear relaxation behind the shock front in 110 molybdenum - from the atomic scale to continuous dislocation fields. Comput. Mater. Science, 149:125 - 133, 2018.

[40] X. Liu, T. Mashimo, K. Ogata, T. Kinoshita, T. Sekine, X. Zhou, and W. J. Nellis. Anomalous elastic-plastic transition of MgO under shock compression. J. Appl. Phys., 114(24):243511, 2013.

[41] B. T. Wang, J. L. Shao, G. C. Zhang, W. D. Li, and P. Zhang. Molecular dynamics simulations of hcp/fcc nucleation and growth in bcc iron driven by uniaxial compression. Journal of Physics: Condensed Matter, 21(49):495702, 2009.

[42] James A. Hawreliak, Bassem El-Dasher, Hector Lorenzana, Giles Kimminau, Andrew Higginbotham, Bob Nagler, Sam M. Vinko, William J. Murphy, Thomas Whitcher, Justin S. Wark, Steve Rothman, and Nigel Park. In situ x-ray diffraction measurements of the $c / a$ ratio in the high-pressure $\epsilon$ phase of shock-compressed polycrystalline iron. Phys. Rev. B, 83:144114.

[43] L. A. Zepeda-Ruiz, A. Stukowski, T. Oppelstrup, and V. V. Bulatov. Probing the limits of metal plasticity with molecular dynamics simulations. Nature, 550:492, 2017.

[44] J. Hawreliak, J. D. Colvin, J. H. Eggert, D. H. Kalantar, H. E. Lorenzana, J. S. Stölken, H. M. Davies, T. C. Germann, B. L. Holian, K. Kadau, P. S. Lomdahl, A. Higginbotham, K. Rosolankova, J. Sheppard, and J.S. Wark. Analysis of the X-ray diffraction signal for the $\alpha-\varepsilon$ transition in shock-compressed iron: Simulation and experiment. Phys. Rev. B, 74:184107, 2006.

[45] X. Cui, W. Zhu, H. He, X. Deng, and Y. Li. Phase transformation of iron under shock compression: Effects of voids and shear stress. Phys. Rev. B, 78:024115, 2008.

[46] J. L. Shao, S. Q. Duan, A. M. He, P. Wang, and C. S. Qin. Microscopic dynamics of structural transition in iron with a nanovoid under shock loading. J. Phys.: Condens. Matter, 22(35):355403, 2010.

[47] C.J. Ruestes, E.M. Bringa, A. Stukowski, J.F. Rodríguez Nieva, Y. Tang, and M.A. Meyers. Plastic deformation of a porous bcc metal containing nanometer sized voids. Comput. Mater. Sci., 88:92 - 102, 2014.

[48] D.A. Porter and K.E. Easterling. Phase Transformations in Metals and Alloys. Springer, B.Y., 1992.

[49] M. Avrami. Kinetics of phase change. I general theory. J. Chem. Phys., 7:1103-1112, 1939.

[50] J. Marian, W. Cai, and V. V. Bulatov. Dynamic transitions from smooth to rough to twinning in dislocation motion. Nature Materials, 3:158, 2004.

[51] On the stress discrepancy at low-temperatures in pure iron. Acta Materialia, 62:267 - 275. 
[52] D. Caillard. Tem in situ straining experiments in fe at low temperature. Philosophical Magazine Letters, 89(8):517-526.

[53] Growth and collapse of nanovoids in tantalum monocrystals. Acta Materialia, 59(4):1354 - 1372.

[54] Probing the character of ultra-fast dislocations. Scientific Reports, 5:16892, 2015.

[55] Stochastic simulation of dislocation glide in tantalum and ta-based alloys. Journal of the Mechanics and Physics of Solids, 53(6):1223 - 1247.

[56] The glide of screw dislocations in bcc fe: Atomistic static and dynamic simulations. Acta Materialia, 54(13):3407 - 3416 .

[57] M. A. Meyers and L. E. Murr. Defect generation in shock wave deformation. In Marc A. Meyers and Lawrence E. Murr, editors, Shock Waves and High-Strain-Rate Phenomena in Metals, pages 487-530. 1980. pp: 516.

[58] M. A. Meyers. Dynamic behavior of materials. John Wiley and Sons, New York, 1994. pp: 425.

[59] M. Bastea, S. Bastea, and R. Becker. High pressure phase transformation in iron under fast compression. Appl. Phys. Let., 95:241911, 2009.

[60] R. Ravelo, T. C. Germann, O. Guerrero, Q. An, and B. L. Holian. Shock-induced plasticity in tantalum single crystals: Interatomic potentials and large-scale molecular-dynamics simulations. Phys. Rev. B, 88:134101, 2013.

[61] D. B. Hayes. Polymorphic phase transformation rates in shock-loaded potassium chloride. Journal of Applied Physics, 45(3):1208-1217.

[62] X. Liu, T. Mashimo, N. Kawai, T. Sano, and X. Zhou. Isotropic phase transition of single-crystal iron (Fe) under shock compression. J. Appl. Phys., 124(21):215101, 2018.

[63] L. Dezerald, D. Rodney, E. Clouet, L. Ventelon, and F. Willaime. Plastic anisotropy and dislocation trajectory in bcc metals. Nature Communications, 7:11695, 2016.

[64] M.S. Duesbery and V. Vitek. Plastic anisotropy in b.c.c. transition metals. Acta Materialia, 46(5):1481 - 1492, 1998. 